CLINICAL STUDY

\title{
Long-term neurodevelopmental outcome in conservatively treated congenital hyperinsulinism
}

\author{
K Mazor-Aronovitch, D Gillis, ${ }^{1}$, D Lobel ${ }^{2}$, H J Hirsch ${ }^{3}$, O Pinhas-Hamiel, D Modan-Moses, B Glaser ${ }^{1}$ and H Landau \\ Pediatric Endocrine Unit, Chaim Sheba Medical Center, Safra Children's Hospital, Tel Hashomer 52621, Israel, ${ }^{1}$ Pediatric Endocrine and Metabolic \\ Endocrine Units, Hadassah-Hebrew University Hospital, Jerusalem, Israel, ${ }^{2}$ Department of Pediatric Neurology, Schneider's Children Medical Center, Petah \\ Tiqva, Israel and ${ }^{3}$ Department of Pediatrics, Shaare Zedek Medical Center, Jerusalem, Israel \\ (Correspondence should be addressed to K Mazor-Aronovitch; Email: kineret@gmail.com)
}

K Mazor-Aronovitch and D Gillis contributed equally to this work

\begin{abstract}
Background: Congenital hyperinsulinism $(\mathrm{CH})$ is treated surgically in many centers (near-total and partial pancreatectomy for diffuse and focal disease respectively). Most patients treated with near-total pancreatectomy developed diabetes during childhood/puberty. $\mathrm{CH}$ patients are at increased risk of neurodevelopmental disorders, some being severe, which are reported to occur in $14-44 \%$ of patients from highly heterogenous cohorts. Over the last few decades, we have treated children with $\mathrm{CH}$ conservatively without surgery. The aim of this study was to assess the neurodevelopmental outcome of these patients.

Design and methods: The study included 21 Ashkenazi CH medically treated patients: 11 homozygotes (diffuse disease) and 9 heterozygotes with mutations on the paternal allele (presumed focal disease). The mean age was 13.7 years (range 8-23). Neurodevelopmental outcomes were assessed by telephone interviews of parents, using a standard questionnaire. Closest age siblings of $\mathrm{CH}$ patients served as controls.

Results: Ten CH patients had perinatal seizures of short duration. Four had post-neonatal seizures, which remitted entirely. During early childhood, four patients (19\%) had hypotonia, eight (38\%) had fine motor problems, seven (33\%) had gross motor problems (clumsiness), and one had mild cerebral palsy. Three patients $(14 \%)$ had speech problems. Eight patients required developmental therapy, compared to one in the control group. Most of these problems were resolved by age 4-5 years. At school age, all were enrolled in regular education, some excelled in their studies, 6 out of 21 patients $(29 \%)$ had learning problems ( 2 out of 21 controls). None had overt diabetes.

Conclusions: Good neurodevelopmental outcome was observed in our conservatively treated $\mathrm{CH}$ patients, with no diabetes as reported in patients undergoing pancreatectomy.
\end{abstract}

European Journal of Endocrinology 157 491-497

\section{Introduction}

The term congenital hyperinsulinism $(\mathrm{CH})$ describes a group of genetic disorders characterized by inadequate suppression of insulin secretion in the presence of recurrent hypoglycemia. It is the most common cause of persistent hypoglycemia in infancy. The incidence has been estimated to be about 1/50 000-1/27 000 live births, but in isolated European communities, such as in parts of Finland, the incidence is much higher (1-3). A very high incidence of $1 / 2675$ has been reported from Saudi Arabia in a community with a high rate of consanguinity.

The paper was partially presented at a symposium of hyperinsulinism at Philadelphia, June 2006, and as a poster at the ESPE meeting, Rotterdam, June-July 2006.
The most frequent form of $\mathrm{CH}$ is associated with recessive mutations of the ATP-sensitive potassium channel KATP. This channel is encoded by two adjacent genes on chromosome 11p15.1, SUR1 (ABCC8), and kir 6.2 (KCNJ11). Two SUR1 (ABCC8) mutations, i.e. $\Delta \mathrm{F} 1388$ and 3992-9g $>\mathrm{a}$, appear to account for more than $90 \%$ of cases in the Ashkenazi Jewish population (4).

Two subtypes of $\mathrm{CH}$ have been described: 1) a diffuse form of the disease manifested as diffuse $\beta$-cell hyperfunction in children with two mutant KATP alleles, and 2) a focal form manifested as small discrete areas of $\beta$-cell adenomatosis, surrounded by normally functioning islets. These children have a paternally derived KATP mutation and loss of maternal 11p15 allele restricted to the pancreatic lesion $(5,6)$.

Recent studies $(7,8)$ analyzed the patterns of acute insulin responses (AIRs) to several secretagogues (calcium, tolbutamine, glucose, and leucine) in infants 
with diffuse and focal $\mathrm{CH}$ with known mutations in the SUR1 or Kir6.2 genes. The results indicate that recessive mutations of the KATP channel genes can be associated with a range of residual channel functions.

In Ashkenazi Jewish patients, $\mathrm{CH}$ is most often associated with null mutations resulting in nonfunctional potassium channels. Affected patients were non-responsive to diazoxide, a channel agonist, and had AIRs to leucine and to the channel antagonist tolbutamide $(7,9,10)$.

Despite the new knowledge of the pathogenesis and genetics of the disease, the management still presents pediatricians with a choice of unsatisfactory treatments that have major long-term implications for the child and his/her family. Today, surgery (partial pancreatectomy for focal disease and near-total pancreatectomy for diffuse disease (DD)) is recommended for children who are resistant to diazoxide and octreotide. Since it became evident that near-total pancreatectomy in patients with DD often failed to cure the hypoglycemia and led to diabetes mellitus by puberty (11), we pioneered a more conservative approach to treating these infants. As we have previously reported, our approach is to maintain normoglycemia using medications (octreotide, diazoxide, and glucagons) together with frequent or continuous feedings using a gastrostomy tube (12-14). Diabetes has thus far not been described in patients with focal disease after partial pancreatectomy. Recently, a dominant heterozygous missense mutation (E1506K) in the SUR ABCC8 gene in a large Finnish family has been reported to cause a mild form of $\mathrm{CH}$ responsive to diazoxide in infancy. In early adulthood, these individuals have glucose intolerance and they go on to develop diabetes mellitus in middle age $(15,16)$. Glucose intolerance has been described in medically treated patients by our group $(11,17)$. However, none of our patients has developed overt diabetes mellitus during follow-up for up to 23 years.

$\mathrm{CH}$ patients exposed to recurrent hypoglycemic episodes are at increased risk of developmental disorders. Psychomotor retardation, learning disabilities, seizures, and other neurological sequelae have been reported to occur in $14-44 \%$ of patients from highly heterogenous cohorts $(18,19)$.

The purpose of the study was to see if patients treated medically had a worse outcome compared with children treated surgically. We describe here the neurodevelopmental outcome of a homogenous group of 21 patients, aged 8-23 years, with the known Ashkenazi mutations, treated aggressively to maintain normoglycemia but without resorting to pancreatectomy.

\section{Subjects and methods}

A total of 31 Ashkenazi patients were treated and followed at Hadassah University Hospital between 1975 and 1997. All patients fulfilled the known criteria for
$\mathrm{CH}$ as described in detail elsewhere (13), i.e. severe, persistent hypoglycemia with non-suppressed insulin levels, inappropriately low free fatty acids and ketone bodies, glycemic response of $>30 \mathrm{mg} / \mathrm{dl}$ to glucagon administration, glucose requirement of over $15 \mathrm{mg} / \mathrm{kg}$ per min in order to maintain euglycemia, and absence of ketone bodies in urine.

Molecular genetic analyses were performed at the time of initial diagnosis in two patients, and retrospectively in the other 29 . Of the 31 patients, 20 were found to have two mutant KATP channel alleles (DD) and 11 had only a paternal mutation. No patients with only a maternal mutation were identified. The patients in whom only a single paternal mutation was identified were presumed to have focal disease (pFD), although it was impossible to prove this histologically in those who did not undergo surgery. All eight patients born before 1982 (six DD and two pFD) had been treated surgically and developed diabetes mellitus during childhood or puberty (11). Since 1982, only 2 of the 23 patients have been treated surgically. The remaining 21 patients, 12 with DD and 9 with pFD, were treated medically, and formed the study group for this report. The decision to attempt medical treatment was based on our observation of patients with $\mathrm{CH}$ who entered remission in infancy (at ages 4 and 11 months) after being scheduled for surgery that had been delayed for various unrelated reasons. Octreotide, which became available in 1986, facilitated our decision to try intensive medical treatment. The choice of therapeutic agents was based on clinical judgment on each patient and included the consideration of the ability of families to cope at home with the treatment regime.

The patients were born in various hospitals and the diagnosis of hypoglycemia was made during the first $24 \mathrm{~h}$ of life in all but one. Our sequential therapeutic strategy is described in detail elsewhere $(12,13)$. At the beginning of treatment, octreotide dose did not exceed $10-15 \mu \mathrm{g} / \mathrm{kg}$ per day. During the following years, it was not necessary to change the dose of octreotide according to weight, thus octreotide per $\mathrm{kg}$ diminished gradually. After 6-12 months, when the dose of octreotide reached $3-4 \mu \mathrm{g} / \mathrm{kg}$ per day, and the patient was able to tolerate longer periods between meals, he/she underwent in-house testing including formal overnight fasting. After this procedure, the patients were allowed to fast overnight at home but were asked to follow fasting blood sugars for several further months. After this home observation period, they continued to test their blood sugar only when ill or when their intake was reduced for any other reason, measuring home glucose before every meal. All the patients in the current study have been in clinical remission, defined as the ability to maintain normoglycemia above $60 \mathrm{mg} / \mathrm{dl}$ after an overnight fast without medication, for at least 3 years. Results of longterm conservative treatment of 7 of the 21 patients reported here have been described previously (12). 


\section{Clinical data collection}

Data were collected from medical records and by telephone or personal interviews with a parent. The collection of data from medical records included the information from intensive care units and follow-up in hospital clinics. Particular attention was given to neurological and developmental status, presence or absence of seizures, and referrals to physical, occupational, or speech therapy and special education. Further information was obtained on the patient and on the sibling closest in age, the latter serving as a control. The range of ages between the patients and their controls was 1-6.5 years, with a median of 3 years. A gender match was possible in eight patients and their controls. Only one patient was a single child in the family. Since there is no standard test available for such a wide range of ages, we created a modified neurodevelopmental questionnaire, which was specially designed for the study (available by e-mail upon request). The parent questionnaire was a semistructured interview designed to review identified early developmental problems, history of seizures, developmental therapy, and a detailed interview regarding problems in the areas of learning, attention, behavior and social skills, educational support, educational achievements, and overall parent assessment of function. The same parent completed a standard detailed interview for both the patient and the sibling closest in age. Direct cognitive or psychological testing was not possible since the parents were reluctant to subject their children to potentially stressful evaluations. The research protocol was approved by the Helsinki committee of the Sheba Medical Center.

\section{Statistical analysis}

Categorical data were expressed as numbers (percentages), continuous variables as the mean value \pm s.D. or mean value and range. For group comparison, the $\chi^{2}$ test was used for categorical variables. Student's $t$-test was used to compare between subgroups. $P<0.05$ was considered significant. All statistical analyses were performed by the SAS software version 8.2 (SAS Institute, Inc, Cary, NC, USA).

\section{Results}

Clinical data on patients with DD and pFD are given in Tables 1 and 2 . In the DD group, seven patients were homozygotes for the 3992-9g $>$ a mutation, two patients were homozygotes for the $\Delta \mathrm{F} 1388$ mutation, and three patients were compound heterozygotes (3992-9g $>\mathrm{a} / \Delta \mathrm{F} 1388)$. In the $\mathrm{pFD}$ group, five patients were heterozygotes for the $3992-9 \mathrm{~g}>\mathrm{a}$ mutation and four patients were heterozygotes for the $\Delta \mathrm{F} 1388$ mutation (all had paternal mutations only).
Table 1 Presentation of patients treated medically.

\begin{tabular}{|c|c|c|c|}
\hline & $\begin{array}{l}\text { Diffuse (DD) } \\
\quad(n=12)\end{array}$ & $\begin{array}{l}\text { Presumed focal } \\
\quad(\mathrm{pFD})(n=9)\end{array}$ & $P$ value \\
\hline $\begin{array}{l}\text { Mean gestational } \\
\text { age (weeks) }\end{array}$ & $37.2(33-41)$ & $39(37-41)$ & 0.11 \\
\hline $\begin{array}{l}\text { Mean birth } \\
\text { weight }(\mathrm{g})\end{array}$ & $\begin{array}{c}3915 \\
(2160-5205)\end{array}$ & $\begin{array}{c}3760 \\
(2450-4550)\end{array}$ & 0.67 \\
\hline $\begin{array}{l}\text { Mean birth } \\
\text { weight } \\
\text { percentile }\end{array}$ & $85 \pm 17$ & $68 \pm 27$ & 0.11 \\
\hline $\begin{array}{l}\text { Hypoglycemia } \\
\text { within first } 48 \mathrm{~h}\end{array}$ & $11 / 12$ & $9 / 9$ & 0.37 \\
\hline $\begin{array}{l}\text { Presenting } \\
\text { symptoms, } \\
\text { seizures }\end{array}$ & $2(17 \%)$ & $3(33 \%)$ & 0.37 \\
\hline
\end{tabular}

All the patients in both groups presented at birth, with the exception of one patient with DD, who first developed hypoglycemia only at the age of 4 months (he was followed since birth because his elder sister had already been diagnosed with $\mathrm{CH}$ ). Mean birth weight, mean percentile for weight, and mean gestational age were similar in both groups.

Eight patients with DD and five patients with pFD were treated with continuous s.c. infusion of octreotide (using a Minimed insulin pump). Four patients with DD and three patients with pFD responded to treatment with diazoxide. All the patients were given frequent feedings during the first year of life (roughly every $2 \mathrm{~h}$ ) and were gradually moved to a normal feeding schedule. Most of the patients had feeding problems ranging from poor sucking and swallowing to retching, frequent vomiting, and refusal of food. In six patients with DD and two patients with pFD, a percutaneous gastrostomy was needed because of the severity of the feeding problems. These patients were also given continuous nocturnal feeding during the first year of life. Thereafter, raw cornstarch was given with the last evening meal to all the patients. In children with a gastrostomy, the establishment of normal feeding patterns was delayed until the age of 3-4 years. Out of the 12 patients with DD, five are part of four nuclear families with more than one child affected. Only one patient with pFD had a family history of $\mathrm{CH}$. There were no significant clinical differences between patients with DD and pFD and between patients with different

Table 2 Clinical course of the patients.

\begin{tabular}{lccl}
\hline & $\begin{array}{c}\text { Diffuse (DD) } \\
(n=12)\end{array}$ & $\begin{array}{c}\text { Presumed focal } \\
(\mathrm{pFD})(n=9)\end{array}$ & $\boldsymbol{P}$ value \\
\hline Diazoxide only & 4 & 2 & 0.58 \\
Octreotide & 8 & 5 & 0.6 \\
Gastrostomy & 6 & 2 & 0.19 \\
$\begin{array}{l}\text { Feeding problems } \\
\text { Clinical remission } \\
\quad \text { age (years) }\end{array}$ & 5 & 5 & 0.6 \\
& $5(1.5-12)$ & $1.7(0.3-5)$ & 0.04 \\
\hline
\end{tabular}

${ }^{\mathrm{a}}$ These data are presented as median + range. 
Ashkenazi mutations. Clinical remission occurred in all patients and was significantly earlier in patients with pFD (two patients entered remission at ages 4 and 11 months). In four patients with DD, occasional, isolated documented hypoglycemic episodes occurred at home, years after all medications were withdrawn. These episodes occurred during acute gastroenteritis in one patient and after introducing a strict regime of diet and exercise for obesity in another. In the remaining two patients, no clear cause for the hypoglycemia was evident.

Patients' heights in both groups are appropriate for parental target height, except for one short patient with DD. One of the patients with DD and two with FD are obese and four patients with DD are overweight. In three of the patients, there is a family history of obesity.

\section{Neurodevelopmental outcome}

Similar results were found in DD and pFD, therefore both groups will be presented together. Ten patients $(48 \%)$ had neonatal seizures, all of which were due to documented hypoglycemia. In eight patients, the seizures occurred only during the first $24 \mathrm{~h}$ after birth, were of short duration, were treated immediately, and did not recur thereafter. (All of them were admitted to the neonatal intensive care unit and had umbilical lines.) Two patients with DD had seizures of short duration after $24 \mathrm{~h}$ of age (one at 2 days of age and the other at 1 week of age, due to a technical problem with the line through which the patient received glucose). Seizures beyond the neonatal period were reported in four patients $(19 \%)$. In two of these patients, the seizures were clearly associated with hypoglycemia. (Those patients were discharged after birth with a diagnosis of transient hypoglycemia of newborn and were re-admitted at the age of 3 months after a convulsion associated with hypoglycemia). Of the other two, one patient had a single febrile seizure and one patient had several seizures during the first year of life, but hypoglycemia was not documented on all occasions. All seizures remitted entirely. At their last follow-up, none of the patients were under treatment with anti-convulsive medication, and none had any evidence of seizure activity for at least 14 years.

Motor and speech problems in infancy and early childhood are presented in Table 3. The $\mathrm{CH}$ patients received more developmental treatment (physical, occupational, and speech and language therapy) compared to the control group (eight patients compared to one control); however, most of these problems were mild. The most prevalent difficulty was motor coordination disorder. Most of the gross motor problems improved by school age. One child had mild right hemiparesis involving primarily the leg without functional limitations and has always attended regular education.
Table 3 Neurodevelopmental characteristics and school performance in patients and controls.

\begin{tabular}{|c|c|c|c|}
\hline & CH patients $(21)$ & Controls (20) & $P$ value \\
\hline \multicolumn{4}{|c|}{ Motor and speech problems in infancy/early childhood } \\
\hline Hypotonia & $4(19 \%)$ & $2(10 \%)$ & 0.4 \\
\hline $\begin{array}{l}\text { Fine motor } \\
\text { problems }\end{array}$ & $8(38 \%)$ & $6(30 \%)$ & 0.58 \\
\hline $\begin{array}{l}\text { Speech } \\
\text { problems }\end{array}$ & $3(14 \%)$ & $1(5 \%)$ & 0.31 \\
\hline $\begin{array}{l}\text { Gross motor } \\
\text { problems }\end{array}$ & $8(38 \%)$ & $2(10 \%)$ & 0.036 \\
\hline CP-mild & $1(5 \%)$ & None & 0.32 \\
\hline \multicolumn{4}{|c|}{ Difficulties at school age } \\
\hline $\begin{array}{l}\text { Learning } \\
\text { difficulties }\end{array}$ & $6(29 \%)$ & $2(10 \%)$ & 0.13 \\
\hline $\begin{array}{l}\text { Attention and } \\
\text { hyperactivity }\end{array}$ & None & $3(15 \%)$ & 0.07 \\
\hline Social skills & $4(19 \%)$ & $2(10 \%)$ & 0.41 \\
\hline Behavior & $2(10 \%)$ & $4(20 \%)$ & 0.34 \\
\hline
\end{tabular}

*Statistically significant $(P<0.05)$.

At school age, none of the patients had mental retardation and all were enrolled in regular education classes. One child with DD was in an advanced program for gifted children. Difficulties at school age are presented in Table 3.

At the end of the questionnaire, parents were asked whether their child had any functional problem that caused problems in their daily activity when compared with their peers. Out of the 21 parents, 3 mentioned one of the following problems each: learning disorder, clumsiness, and emotional problems.

Fifteen of these children are currently being followed personally by several of the authors (G D, H J H, G B, and $\mathrm{L} \mathrm{H}$ ) and all are normally emotional, respond normally to questions, and have no neuropsychological damage as per clinical examination.

\section{Discussion}

In this study, we describe the long-term outcome of a group of Ashkenazi patients with known mutations in the SUR 1 gene. It is most likely that in other centers, the majority of these patients would have undergone surgery, as did all the eight Ashkenazi patients treated by us before 1982. All of our patients who had undergone pancreatectomy subsequently developed diabetes mellitus.

It is noteworthy that most of the patients were treated surgically or medically long before their mutations were known. There is a great variability in the severity of disease between patients with the same mutations and between affected siblings with DD within the same family. In three families with two or three affected children each, one patient in each family responded to treatment with diazoxide, whereas their siblings failed to respond to the same medical treatment. Based on 
a study involving seven patients, Henwood et al. classified both Ashkenazi mutations as null mutations, causing totally non-functional KATP channels (7). Our data on these three families show that this is not always the case, since some of our patients carrying the 3992$9 \mathrm{~g}>\mathrm{a}$ mutation had milder disease and responded well to diazoxide.

The medically treated patients reported here showed a more favorable neurodevelopmental outcome compared with our ten patients treated surgically. The neurobehavioral profiles of seven of these patients at school age operated before 1982 (five with DD and two with FD, whose genetic analyses were done many years later) have been reported: one patient has subnormal intelligence, four have epilepsy, and all of them have some sort of learning disability (20). Although these patients were treated aggressively since birth, our treatment protocol since then has considerably improved, especially since octreotide became available.

Most of our patients came from large families with children close in age to each other. Therefore, the best control possible in our opinion was the sibling closest in age. Since all patients and siblings underwent genetic testing, the control siblings were definitely unaffected.

It is very difficult to compare the neurodevelopment of our patients with others reported in the literature, because most series present data from highly heterogeneous cohorts who were diagnosed without the benefit of molecular genetic analysis and who were treated with different combinations of medications, surgery, and feeding regimens.

The outcome of our patients is comparable with that of patients with early onset of the disease, who underwent pancreatectomy, described recently by three groups from specialized centers $(18,19,21)$. Menni et al. (18) described $90 \mathrm{CH}$ patients treated between 1982 and 1998 and followed for at least 10 years. Twenty-seven patients were treated medically with diazoxide and 63 patients underwent surgery. Twenty-six percent of the patients had psychomotor retardation including $8 \%$ with major retardation (more prevalent in operated patients) and 18\% with intermediate disability (no difference between the two groups). Eighteen percent of patients had epilepsy, with no difference between the two groups. Meissner et al. (19) described $114 \mathrm{CH}$ patients born between 1975 and 2002. The mean age was 14 years. Fortyseven patients responded to diazoxide, two patients were treated with octreotide, and the others were treated surgically. Forty-four percent of the patients had neurodevelopmental retardation, of whom $18 \%$ were severe and $26 \%$ were mild. Twenty-five percent had epilepsy. There was no description of differences between medical and surgical patients, but they did note that among the patients with psychomotor or mental retardation, $51 \%$ had undergone surgery. Steinkrauss et al. (21) described $68 \mathrm{CH}$ patients treated between 1980 and 2000. Twenty-six patients were treated medically with diazoxide, 35 underwent surgery and 7 patients had transient hyperinsulinism. The median age at the time of analysis was 5 years for the medically treated patients and 7 years for the surgical patients. One-third of the patients had developmental delay, and this was more prevalent in the surgical group. Twenty-three percent of the surgical patients had a very low psychological performance compared with $4 \%$ in the medically treated patients. Nine percent of the surgical patients had low to low-moderate performance compared with $23 \%$ in the medically treated patients. Thirty-four percent of the cohort required special education, but $75 \%$ of those in special education had undergone pancreatic surgery. There were no differences in the outcome between patients with DD or $\mathrm{pFD}$ as was also observed by others (22). In conclusion, 26-44\% of the patients in the mentioned papers manifested some developmental delay, but, as in our series, most patients recovered later. In our study, there seems to be a particular association between early hypoglycemia and subsequent motor coordination difficulties. More patients received early developmental therapy during preschool years in comparison with their siblings, the most prevalent difficulties being a combination of gross and fine motor problems (five children in the $\mathrm{CH}$ group). Most of these were resolved by school age.

The height of our patients is relevant since octreotide suppresses growth hormone secretion. Interestingly, current height was appropriate for parental height, in all patients including those treated with octreotide. During the first year of octreotide treatment, a deceleration of growth was observed in a few patients. As the dose per $\mathrm{kg}$ of octreotide diminished with time, catch-up growth was evident, except in one patient whose growth continued along the third percentile. We have reported that after an increase in weight percentile during the first months of life, weight gain normalized during treatment and remained normal after the cessation of treatment in most patients (12). The latest data from our questionnaire show a tendency for overweight/obesity, especially in patients with DD, even in the absence of a familial history of obesity. This can be explained by the tendency of parents to overfeed these children for fear of hypoglycemia. Alternatively, these patients may continue to overeat spontaneously because of unnoticed decreases in glucose levels or out of habit nurtured by their parents during infancy.

Until recently, the definitive diagnosis of focal lesions required histologic investigation of resected pancreata. More recently, the successful use of F-fluoro-L-dopa PET scans has made it easier to detect patients with focal lesions (23-25). Since the PET scan was not available for our patients, we used genetic criteria to identify patients most likely to have focal $\mathrm{CH}$. In the Ashkenazi population, more than $90 \%$ of the $\mathrm{CH}$-associated alleles can be identified by simple genetic analysis. We assumed 
that patients having only a single mutation on the paternal allele had focal disease. However, theoretically, they may have harbored a second unidentified mutation on the maternal allele. If a significant number of unidentified mutations were present in this population, one would expect that in about half of the patients with one mutation, this would be located on the maternal allele. In fact, in all 11 patients where only a single mutation was found, it was on the paternal allele. It is highly unlikely that this deviation from the expected distribution occurred by chance $(P=0.03)$, providing evidence that most, if not all of these patients, indeed had focal disease. Since the only significant difference observed between the patients with DD and those with pFD was the age at clinical remission, even if some, or all, of the patients with pFD had in fact DD, the major findings of this study would not be changed. There is a widely accepted consensus that children with FD should undergo partial selective pancreatectomy. Tests of postsurgical glucose metabolism were reported to be normal and these children are considered cured (22). The longterm outcome of these patients into adulthood has not yet been studied.

The retrospective analysis of our patients with a paternal mutation only, and therefore presumed FD, shows that they entered remission significantly earlier than those with DD and most of those studied so far had normal oral glucose tolerance test, normal fasting glucose levels, and normal HbA1C levels (17). These data suggest that conservative treatment of FD patients may be a reasonable alternative; however, until more data on the long-term outcome of conservatively treated patients with definitive diagnosis of FD are available, no conclusions can be drawn.

When comparing conservative treatment of $\mathrm{CH}$ with surgery, particularly for DD patients, one must take into consideration the fact that the conservative method poses a significant challenge to the family, and it is clearly not possible to apply it without highly cooperative parents. One of the most challenging problems is feeding, due to foregut dysmotility and gastroesophageal reflux, the pathophysiology of which is yet to be determined $(1,26)$. Indeed, conservative treatment of $\mathrm{CH}$ is not suitable for all families. However, it is important to allow motivated parents to follow this method. They can now be made aware that after the difficult first year, the condition becomes gradually easier to control, with a slow increase in the time between meals and decrease in the frequency of hypoglycemia. Also, the alternative of treating postpancreatectomy diabetes in infancy and early childhood is only slightly less stressful, and such diabetes is lifelong with multiple potential complications.

There are several important limitations in our study. This is not a randomized prospective study. Historical and school performance data are based on parents' reports which may have not been precise. We cannot rule out that some learning disorders in the $\mathrm{CH}$ group may have been missed or denied by the parents. On the other hand, there is increased awareness regarding potential developmental problems in children with complicated postnatal courses; they are followed closely and might have a higher detection rate for mild developmental delays. The parents might have tended to over-test the other siblings too. Also in Israel, in general, there is a near-total coverage of the population with government-funded well child clinics. (Denver Developmental test is done routinely at least until the age of 1.5 years.) Since nearly all infants and young children undergo psychoneurological evaluation, some, who are diagnosed in infancy as having neurodeveopmental abnormalities, grow up to be completely normal. The number of patients in each group is small and therefore difficult to interpret. Larger, multicenter studies are still needed. Despite these limitations, however, developmental outcome is the major issue. It is clear, based on this study, that if optimal care is provided, the risk of major neurological handicaps, seizures, and need for special schooling will be low in conservatively treated patients. In this regard, it appears that conservative treatment offers patients at least as good an overall long-term functional prognosis as does early pancreatectomy.

\section{Acknowledgement}

We thank the nurse team of the Endocrine Unit at Hadassah-Hebrew University Hospital in Jerusalem, Israel.

\section{References}

1 Aynsley-Green A, Hussain K, Hall J, Saudubray JM, NihoulFekete C \& Lonlay-Debeney P. Practical management of hyperinsulinism in infancy. Archives of Disease in Childhood. Fetaland Neonatal Edition $2000 \mathbf{8 2}$ F98-F107.

2 Otonkoski T, Ammala C, Huopio H, Cote GJ, Chapman J \& Cosgrove K. A point mutation inactivating the sulfonylurea receptor causes the severe form of persistent hyperinsulinemic hypoglycemia of infancy in Finland. Diabetes 1999 $\mathbf{4 8} 408-415$.

3 Mathew PM, Young JM, Abu-Osba YK, Mulhern BD, Hammoudi S \& Hamdan JA. Persistent neonatal hyperinsulinism. Clinical Pediatrics 198827 148-151.

4 Nestorowicz A, Wilson BA, Schoor KP, Inoue H, Glaser B \& Landau $\mathrm{H}$. Mutations in the sulonylurea receptor gene are associated with familial hyperinsulinism in Ashkenazi Jews. Human Molecular Genetics 19965 1813-1822.

5 de Lonlay P, Fournet JC, Rahier J, Gross-Morand MS, PoggiTravert F \& Foussier V. Somatic deletion of the imprinted 11p15 region in sporadic persistent hyperinsulinemic hypoglycemia of infancy is specific of focal adenomatous hyperplasia and endorses partial pancreatectomy. Journal of Clinical Investigation $1997 \mathbf{1 0 0}$ 802-807.

6 Ryan F, Devaney D, Joyce C, Nestorowicz A, Permutt MA \& Glaser B. Hyperinsulinism: molecular aetiology of focal disease. Archives of Disease in Childhood 1998 79 445-447.

7 Henwood MJ, Kelly A, MacMullen C, Bhatia P, Ganguly A \& Thornton PS. Genotype-phenotype correlations in children with 
congenital hyperinsulinism due to recessive mutations of the adenosine triphosphate-sensitive potassium channel genes. Journal of Clinical Endocrinology and Metabolism 200590 789-794.

8 Huopio H, Jaaskelainen J, Komulainen J, Miettinen R, Karkkainen P \& Laakso M. Acute insulin response tests for the differential diagnosis of congenital hyperinsulinism. Journal of Clinical Endocrinology and Metabolism 2002 87 4502-4507.

9 Ferry RJ Jr, Kelly A, Grimberg A, Koo-McCoy S, Shapiro MJ \& Fellows KE. Calcium-stimulated insulin secretion in diffuse and focal forms of congenital hyperinsulinism. Journal of Pediatrics $2000137239-246$.

10 Grimberg A, Ferry RJ Jr, Kelly A, Koo-McCoy S, Polonsky K \& Glaser B. Dysregulation of insulin secretion in children with congenital hyperinsulinism due to sulfonylurea receptor mutations. Diabetes $200150322-328$.

11 Leibowitz G, Glaser B, Higazi AA, Salameh M, Cerasi E \& Landau H. Hyperinsulinemic hypoglycemia of infancy (nesidioblastosis) in clinical remission: high incidence of diabetes mellitus and persistent beta-cell dysfunction at long-term follow-up. Journal of Clinical Endocrinology and Metabolism 199580 386-392.

12 Glaser B, Hirsch HJ \& Landau H. Persistent hyperinsulinemic hypoglycemia of infancy: long-term octreotide treatment without pancreatectomy. Journal of Pediatrics $1993123644-650$.

13 Glaser B, Landau H \& Permutt MA. Neonatal Hyperinsulinism. Trends in Endocrinology and Metabolism 199910 55-61.

14 Glaser B \& Landaw H. Long-term treatment with the somatostatin analogue SMS 201-995: alternative to pancreatectomy in persistent hyperinsulinaemic hypoglycaemia of infancy. Digestion 1990 45 27-35.

15 Reimann F, Huopio H, Dabrowski M, Proks P, Gribble FM \& Laakso M. Characterisation of new KATP-channel mutations associated with congenital hyperinsulinism in the Finnish population. Diabetologia $2003 \mathbf{4 6} 241-249$.

16 Huopio H, Otonkoski T, Vauhkonen I, Reimann F, Ashcroft FM \& Laakso M. A new subtype of autosomal dominant diabetes attributable to a mutation in the gene for sulfonylurea receptor 1. Lancet $2003361301-307$.

17 Gillis D, Landau H, Zangen DH \& Glaser B. Glucose intolerance in patients with persistent hyperinsulinemic hypoglycemia of infancy. Abstract 2086, 82nd annual meeting of the endocrine society, June 2000.
18 Menni F, de Lonlay P, Sevin C, Touati G, Peigne C \& Barbier V. Neurologic outcomes of 90 neonates and infants with persistent hyperinsulinemic hypoglycemia. Pediatrics 2001107 476-479.

19 Meissner T, Wendel U, Burgard P, Schaetzle S \& Mayatepek E. Longterm follow-up of 114 patients with congenital hyperinsulinism. European Journal of Endocrinology 2003 149 43-51.

20 Gross-Tsur V, Shalev RS, Wertman-Elad R, Landau H \& Amir N. Neurobehavioral profile of children with persistent hyperinsulinemic hypoglycemia of infancy. Developmental Neuropsychology $199410153-163$.

21 Steinkrauss L, Lipman TH, Hendell CD, Gerdes M, Thornton PS \& Stanley CA. Effects of hypoglycemia on developmental outcome in children with congenital hyperinsulinism. Journal of Pediatric Nursing 200520 109-118.

22 Giurgea I, Ribeiro MJ, Boddaert N, Touati G, Robert JJ \& Saudubray JM. Congenital hyperinsulinism in newborn and infant. Archives of Pediatrics 200512 1628-1635.

23 Ribeiro MJ, de Lonlay P, Delzescaux T, Boddaert N, Jaubert F \& Bourgeois S. Characterization of hyperinsulinism in infancy assessed with PET and 18F-fluoro-L-DOPA. Journal of Nuclear Medicine $2005 \mathbf{4 6} 560-566$.

24 Otonkoski T, Nanto-Salonen K, Seppanen M, Veijola R, Huopio H \& Hussain K. Noninvasive diagnosis of focal hyperinsulinism of infancy with [18F]-DOPA positron emission tomography. Diabetes $20065513-18$.

25 Mohnike K, Blankenstein O, Christesen HT, De Lonlay J, Hussain K \& Koopmans KP. Proposal for a standardized protocol for $18 \mathrm{~F}$ DOPA-PET $(\mathrm{PET} / \mathrm{CT})$ in congenital hyperinsulinism. Hormone Research $2006 \mathbf{6 6} 40-42$.

26 Lindley KJ, Knafelz D, St Louis D, Dunne MJ, Aynsley-Green A \& Milla PJ. Surface electrogastrographic evidence of foregut dysmotility in persistent hyperinsulinaemic hypoglycaemia of infancy (PHHI). Journal of Physiology 1997499108.

Received 15 May 2007

Accepted 19 July 2007 